\title{
Los centros comerciales como paisajes globales en la reconfiguración de los espacios público-privados*
}

\author{
Luis A. Escudero Gómez \\ Universidad de Castilla-La Mancha. Departamento de Geografía y Ordenación del Territorio \\ luisalfonso.escudero@uclm.es
}

\author{
Andrés Castiblanco Roldán \\ Universidad Distrital Francisco José de Caldas \\ afcastiblancor@udistrital.edu.co
}

Jaime A. Wilches Tinjacá

Institución Universitaria Politécnico Grancolombiano

jwilches@poligran.edu.co

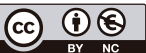

Recibido: septiembre de 2020

Aceptado: abril de 2021

Publicado: septiembre de 2021

\section{Resumen}

Los centros comerciales forman parte de los paisajes globales. Se han transformado en un signo territorial del consumo a escala planetaria y de la mercantilización y apropiación de los espacios públicos. El artículo tiene un objetivo doble: primero, la consideración de los centros comerciales como un paisaje urbano derivado de la globalización y, segundo, el análisis de la dicotomía entre espacios públicos y privados a través de estas áreas particulares de consumo. Es un estudio conceptual que recurre a una extensa revisión literaria científica, con más de 125 obras consultadas y con referencias a autores clave. Además, se ha realizado una práctica de observación. Los principales resultados demuestran que estos complejos son aparentemente públicos, pero con un control, diseño, estructura y finalidad privada basados en el lucro. Se han convertido en áreas de consumo de éxito global y en lugares de relación social. Estos paisajes globales actúan como sustitutos de los espacios públicos urbanos, calles y plazas, por un urbanismo comercial y privado. El artículo resulta de interés para los responsables políticos, los gestores privados de los centros comerciales y los académicos.

Palabras clave: paisaje global; centro comercial; espacio público; privatización

* La realización de este artículo se inscribe en el marco de los proyectos RTI2018-096435-BC22 y RTI2018-093296-B-C22 (MCIU/AEI/FEDER). Los autores quieren agradecer a los revisores anónimos y al consejo editorial la lectura cuidadosa y completa de este manuscrito, y los comentarios reflexivos y las sugerencias constructivas que han ayudado a mejorarlo. Se agradece a Ana María Coll haber acompañado la observación de muchos de los malls. 
Resum. Els centres comercials com a paisatges globals en la reconfiguració dels espais publicoprivats

Els centres comercials formen part dels paisatges globals. S'han transformat en un signe territorial del consum a escala planetària i de la mercantilització i apropiació dels espais públics. L'objectiu principal de l'article és analitzar el centre comercial com un paisatge global en la reconfiguració dels espais publicoprivats. És un estudi conceptual que recorre a una extensa revisió literària científica, amb més de 125 obres consultades i amb referències a autors clau. A més, s'ha portat a terme una pràctica d'observació. Els principals resultats demostren que aquests complexos són aparentment públics, però amb un control, disseny, estructura i finalitat privada basats en el lucre. S'han convertit en àrees de consum d'èxit global i en llocs de relació social. Aquests paisatges globals actuen com a substituts dels espais públics urbans, carrers i places, per un urbanisme comercial i privat. L'article és interessant per als responsables polítics, els gestors privats dels centres comercials i els acadèmics.

Paraules clau: paisatge global; centre comercial; espai públic; privatització

Résumé. Les centres commerciaux en tant que paysages mondiaux dans la reconfiguration des espaces public-privé

Les centres commerciaux font partie des paysages mondiaux. Ils sont devenus un signe territorial de consommation à l'échelle planétaire ainsi que de commercialisation et d'appropriation des espaces publics. L'objectif principal de l'article est d'analyser le centre commercial en tant que paysage global dans la reconfiguration des espaces publics-théoriques. Il s'agit d'une étude conceptuelle qui utilise une vaste revue littéraire scientifique, avec plus de 125 ouvrages consultés et avec des références à des auteurs clés. De plus, une observation a été effectuée. Les principaux résultats montrent que ces complexes sont apparemment publics, mais avec un contrôle, une conception, une structure et un but privés basés sur le profit. Ils sont devenus des zones de consommation à succès mondial et des lieux d'interaction sociale. Ces paysages globaux se substituent aux espaces publics urbains, aux rues et aux places, à l'urbanisme commercial et privé. L'article intéresse les décideurs politiques, les gestionnaires privés de centres commerciaux et les universitaires.

Mots-clés : paysage mondial ; centre commercial ; espace public ; privatisation

Abstract. Shopping centres as global landscapes for the reconfiguration of public-private spaces

Shopping centres are part of what we call "Global Landscapes": common areas in the spatial model, brought about by globalization. They have become a sign of consumerism in a specific territory all over the planet and of commercialization and appropriation of public spaces. The aim of the present text is to analyse the concept of shopping spree as a global icon for the reconfiguration of theoretical public spaces. It is a conceptual study that uses an extensive scientific literary review, with more than 125 studies consulted and with references to key authors. In addition, an observation has been carried out. The most significant results presented here show that these premises are public in appearance, but there is control, design, structure, and private objectives that aim to obtain profit. Their tangible and intangible resources have made them areas for consumerism of worldwide success and a framework for social interaction. These global locations promote commercial and private urban development in replacement of public spaces, streets and squares. The article is of great interest for public managers, private agents in shopping centres, and academics.

Keywords: global landscape; shopping centre; mall; public space; privatization 


\title{
Sumario
}

\author{
1. Introducción 4. Conclusiones \\ 2. Metodología Referencias bibliográficas \\ 3. Resultados
}

\section{Introducción}

«Hay grandes edificios, autopistas, centros comerciales, pero no hay calles».

(Vilas, 2017: 36)

Dentro de las ciencias sociales, la geografía ha encontrado en el paisaje ${ }^{1}$ una unidad clave en las lecturas de los fenómenos territoriales, tanto en los procesos geomórficos (Cosgrove, 2008; Berque, 2009) como en sus correlaciones con lo humano y sus efectos en el medio (Santos, 1988; Berque, 1990; Maderuelo, 2005). En la actualidad, el paisaje es uno de los campos de estudio más importantes de la geografía, y es visto desde diferentes perspectivas (Daniels et al., 2011). En estos enfoques, la relación que prevalece es un estatuto de lectura de las morfologías naturales y artificiales en su encadenamiento temporal. En cierto modo, toda modelación de los lugares obedece a una relación mediotiempo-sujeto, observada con rigurosidad desde los estudios de la trayección de Berque (2009) y puesta en clave de reflexión a partir de diferentes conjuntos analíticos, en los cuales están las mediaciones con el intercambio, el hábitat y el conflicto (Nogué, 2007).

El paisaje posiciona un orden de la percepción de las sociedades sobre sus influjos espaciales. Cada unidad da cuenta del acervo social e histórico que consolida los lugares y su evolución morfológica en torno a lenguajes, materialidades y memorias que muestran los ritmos, mentalidades e imaginarios con los que las ciudades, regiones y países se dinamizan. Sin embargo, frente a esta singularidad territorial, la globalización ha impuesto un modelo espacial planetario con unos lugares comunes globales.

Se podría proponer que la globalización constituye un sistema de organización espacial de escala planetaria que configura el panorama actual, estudiado en su mayoría con acento en enfoques de corte economicista y sociológico (Bauman, 2000; Ortiz, 2004; Giddens, 2007). Estos estudios ubicaron el apogeo o emergencia de la globalización con la internacionalización económica

1. En el presente artículo se entiende el término paisaje según la definición del Convenio

Europeo del Paisaje de 2000: «Cualquier parte del territorio tal como la percibe la población, cuyo carácter sea el resultado de la acción y la interacción de factores naturales y/o humanos» (Consejo de Europa, 2000). 
y las dos últimas revoluciones industriales, lo cual, en resumen, requirió el nacimiento de su crítica reflexiva (Baricco, 1996) y la consecuente resonancia geográfica (Santos, 1994, 1997).

Esta serie de fenómenos deja a su paso la creación de vocablos espaciales como lo global y lo local, que cobran fuerza con la emergencia de espacios que dan cuenta de los intercambios sociales. En ellos, una lógica de apropiación y modelación del territorio se torna en un signo común en la ocupación y el uso del espacio territorial. A partir de este fenómeno, se propone la categoría de "paisajes globales» (Castiblanco, 2018) para definir aquellos espacios que se han diseñado en clave planetaria para representar la presencia de una institución social antigua e instrumental de las relaciones sociales: el mercado. Las relaciones que se institucionalizan en su agenciamiento condicionan la creación y la transformación territorial en las sociedades. Los paisajes globales son los lugares comunes del modelo espacial planetario de la globalización.

El mercado como institución prepara las coyunturas que dan sentido a la producción social de los espacios de intercambio. En este último caso, el mall o el shopping center, el centro comercial, se ha transformado en un signo territorial del intercambio a escala planetaria. Sassano (2015: 409) los considera como «los máximos espacios de consumo simbólico y mercantil de la sociedad capitalista actual». Se trata de un tipo arquitectónico global que se mueve a través de las fronteras nacionales y culturales en la corriente de la globalización neoliberal. No obstante, manteniendo su carácter de paisajes globales, su realidad tiene también una expresión temporal y geográfica resultado de las negociaciones entre los desarrolladores de centros comerciales — representantes de una lógica global de acumulación capitalista-y los actores locales - arquitectos/gobiernos/ciudadanos- (Gosseye y Avermaete, 2019).

La noción de vitrina de origen moderno se revoluciona con la instalación de estos enclaves espaciales del comercio minorista a lo largo y ancho del planeta a modo de escaparates universales que, en clave de marcas, se posicionan sobre las cotidianidades de urbes tan disímiles como Bogotá, Hong Kong y París, por ejemplo. Son caricaturas del consumo, espacios replicados, anómicos, inauténticos, controlados y segregados para un imaginario burgués de un espacio social aséptico libre de pobreza, crimen y miedo (Simone, 2019). Los centros comerciales crean atmósferas de ocupación espacial que se presentan como oasis transterritoriales donde los ciudadanos del mundo se pueden orientar en sociedades distantes y particulares. Como paisajes globales, ofrecen la posibilidad de permanente conexión e igualdad, por lo menos en su ensueño, con el mundo cosmopolita, lo que no implica que su presencia genere o potencie todo lo contrario, el revés de la marca (Klein, 1999; Castiblanco, 2015) o de los circuitos globales del capital (Sassen, 1991), en los cuales la cara iluminada del aviso esconde la oscuridad de las relaciones que potencian la presencia de ciudadanos del mundo sobre ciudadanos locales oprimidos, desiguales en últimas, desconectados (García, 2004).

La comprensión del paisaje global pasa por la fractura y recomposición de sus espacios. Por eso es necesario entender la manera en que se reconfigura 
el espacio público como escenario permanente de lucha social y de posicionamiento sobre lo que algunos autores han propuesto como el derecho a la ciudad (Lefebvre, 1968; Harvey, 2008). Tal representación de igualdades y posibilidades se debate sobre la presencia de la propiedad privada como signo de la restricción y de la negación de derechos colectivos sobre el disfrute de los espacios.

¿Suponen los centros comerciales una apropiación privada de los espacios públicos urbanos a escala global? Respondiendo a esta pregunta principal de investigación, el artículo tiene un objetivo doble: primero, la consideración de los centros comerciales como un paisaje urbano derivado de la globalización y, segundo, el análisis de la dicotomía entres espacios públicos y privados a través de estas áreas particulares de consumo. Estos complejos forman una realidad complicada y heterogénea que hay que estudiar para entender la influencia que ejercen sobre el resto de los espacios urbanos (Valdelvira, 2010). La investigación supone la novedad de entender los paisajes globales y la actual reconfiguración de los espacios público-privados a través de los centros comerciales, referentes vectores de este proceso. El estudio es una aportación teórica, conceptual y reflexiva que se inserta en los debates científicos sobre el espacio público y, por lo tanto, su aportación principal es académica. Es de interés académico y, al mismo tiempo, de utilidad para los responsables políticos y los gestores privados de los centros comerciales.

La estructuración del texto propone como punto de partida la noción experimental de paisajes globales como la categoría con la cual el modelo del centro comercial y su lenguaje espacial desarrolla un proceso de contaminación mundial. Como señala Ortiz (2004), y a partir del clásico trabajo de Ritzer (1994), dentro de una McDonalización de las economías, los sujetos, al sentirse perdidos en las particularidades de las culturas y sus sociedades, visitan y consumen en los centros comerciales la afabilidad del hogar. De ahí la estrategia de una familiaridad transnacional o transterritorial que se puede proponer como paisaje común del capitalismo contemporáneo. Este carácter global de estos enclaves geográficos se explica en la restructuración de lo público y lo privado como formas de innovación territorial, con la cual se dinamizan sinergias a la vez que se tejen nuevas desigualdades.

Se propone un orden en los apartados que parte de esta introducción y sigue con una referencia a la metodología empleada. A continuación, se desarrollan los resultados para acabar con unas conclusiones.

\section{Metodología}

El artículo es un estudio conceptual que recurre a una extensa revisión literaria científica, con más de 125 obras consultadas y con referencias a autores clave. Además, se ha realizado un trabajo de campo. De esta forma, la metodología del artículo mezcla los tipos de observación indicados por Higueras (2003), es decir, la observación directa o sensorial, mediante el trabajo de campo, y la observación indirecta, documental o diferida, mediante la revisión literaria. 
Este tipo de estrategia constituye un dispositivo metodológico sistemático en el sentido de desarrollar constantemente una condición analítica-semiótica espacial observership (Cobley, 2017), que permite correlatar las fuentes documentales con las percepciones que experimentan el tamiz de la mirada categorial, al tiempo que la experiencia social y territorial.

Se recurre a la literatura científica disponible mediante una exploración de trabajos seleccionados. Al no tratarse estrictamente de un artículo sistémico de revisión literaria sino de un estudio conceptual, y dado que la lectura de todas las publicaciones que abordan las cuestiones tratadas en la investigación es imposible por su elevada dimensión, se realizó una elección de obras, con referencias a la literatura relevante de las ciencias sociales, con autores clave como Augé, Bauman, Berque, Castells, Delgado, Gehl, Giddens, Harvey, Klein, Lefebvre, Ritzer, etcétera, y a investigaciones previas sobre los centros comerciales y los espacios públicos que auxiliaban el entendimiento del objeto de estudio. Este estado del arte se ha organizado en base a los temas clave del artículo: los paisajes globales, los centros comerciales y los espacios públicos y su privatización. Especialmente, se ha llevado a cabo una búsqueda bibliográfica de los trabajos anteriores significativos que han abordado la cuestión de cómo los centros comerciales suponen una suplantación de los espacios públicos urbanos.

Por otro lado, el trabajo de campo se fundamenta en las experiencias previas de los autores en los centros comerciales en diferentes lugares del mundo y en la reflexión de lo observado. Como señalan Kitchin y Tate (2000), el método de observación se basa en la capacidad del observador para interpretar lo que está sucediendo y por qué. Desde hace más de una década, los investigadores vienen observando los centros comerciales en diferentes lugares del mundo. De este trabajo, se han recuperado las notas de campo relacionadas con la sustitución de los espacios públicos que ejercen estos complejos. Con ese interés, se han usado observaciones realizadas en 2018 en el país originario de los centros comerciales, los Estados Unidos, en el primer centro comercial que se creó y que todavía sigue abierto, Southdale Center, en Edina, y en el complejo de mayor tamaño, el Mall of America en Mineápolis, ambos en el estado de Minesota, y en el Sureste Asiático en 2019, en concreto en Singapur, donde los centros comerciales han alcanzado un gran desarrollo. Con este trabajo de campo en lugares tan distanciados, se lograba evidenciar la realidad de los centros comerciales como paisajes globales. Asimismo, se han llevado a cabo observaciones más continuadas en el centro comercial La Vaguada de Madrid y en el de Ciudad Tunal de Bogotá, en los años 2019-2020, para aportar dos casos significativos concretos de apropiación de los espacios públicos en áreas urbanas residenciales por parte de estos establecimientos. No obstante, al igual que en el caso de Torabi et al. (2021), el artículo no presenta un estudio de caso comparativo tradicional, sino que utiliza los casos para ilustrar su marco conceptual.

Finalmente, el trabajo intenta combinar lo universal y lo particular siguiendo el método dialéctico de Harvey, en el que la teoría y el análisis de situaciones concretas se alimentan mutuamente (Benach y Albet, 2019). 


\section{Resultados}

En este apartado se parte de los debates sobre el espacio público y su relación con los centros comerciales. Después, se estudian las tipologías de los espacios públicos y el difícil encajamiento de los paisajes globales de los centros comerciales. A continuación, se trata el paisaje global-centro comercial como áreas de consumo de éxito global y lugares de relación social. Finalmente, se profundiza sobre cómo han sustituido los espacios urbanos públicos, sus calles y plazas, por un urbanismo comercial privado.

\subsection{El centro comercial como paisaje global: debates sobre el espacio público}

El espacio congrega la naturaleza y lo humano en una trama de actos y relatos y formas que consolidan usos e interiorizaciones en las comunidades a través de sus modos de habitar. Un hábitat es una entelequia implicativa del cúmulo de saberes técnicos de los que se vale un grupo para existir.

Desde tal correspondencia, la interrogación por el espacio y las grafías del morar - entendidas a través de la noción de habitar que nos da Heidegger (1994) como la manera en que los mortales son en la tierra-, del modo en que este lazo influye y permite dar paso a la relación entre hábitat y paisaje, insinúa cuestionar el itinerario de la cultura por los ingredientes que congregan el diseño o la transformación de los territorios. El paisajismo y el hábitat, elementos esenciales en los cuales las sociedades asientan su programa de establecimiento y perfeccionamiento, son verdaderamente locuciones de un aforo ideacional de los pueblos para constituir y programar un régimen artificial de ocupación social de varios colectivos establecidos.

Concebir dichas técnicas de modelación de territorios que involucran esferas íntimas y sociales lleva por consecuencia a la alusión sobre el peso de la construcción/producción social del espacio-ciudad (Lefebvre, 1974; Harvey, 2008). Este hecho invoca la reflexión de las tensiones entre lo público y lo privado que se materializan en la construcción urbanística, estética y ciudadana de los espacios humanizados.

El espacio público en las ciudades es un tema atractivo para múltiples académicos de diversas disciplinas, desde el urbanismo (Borja y Muxí, 2003) y la antropología y la sociología (Castells, 1971; Augé, 1996, Serje y Salcedo, 2008) hasta la geografía (García, 2011; Estévez, 2012; Tomé, 2014; Qian, 2020). De manera particular, es un tema de análisis clave dentro de los estudios urbanos dado que el espacio público es una categoría básica para entender la ciudad (Carrillo-Moedano, 2019). El significado y la función del espacio público están en el centro de un debate vibrante (Tchoukaleyska et al., 2019). De hecho, la literatura sobre el tema es inabarcable, aunque obras como las colectivas de Carr et al. (1992) y de Carmona et al. (2012) facilitan el acceso general a sus conceptos básicos.

¿Qué es un espacio público? Como suele suceder en la definición de conceptos complejos en las ciencias sociales, la respuesta no es sencilla, ni tampoco 
única y universal (Koohsari et al., 2015). De hecho, Staeheli y Mitchell (2007) argumentan que precisamente esta ambigüedad es un potencial problema para poder distinguir entre lo público y lo privado. Existen varias definiciones de espacio público que se distinguen por cuestiones de propiedad, control, acceso y uso. La acepción jurídica es la más evidente: espacio de titularidad pública, es decir, propiedad del estado, y sobre el cual solo el estado tiene autoridad (Delgado, 2011). Madanipour (1996: 144) lo define como el «espacio que no está controlado por individuos u organizaciones privadas y, por lo tanto, está abierto al público en general». Otros autores se refieren a lugar «de acceso público donde las personas van para actividades grupales o individuales» (Carr et al., 1992: 50). En definitiva, como afirma Bauman (2000), es un espacio donde la gente puede compartir como persona pública. Es el área que es de todos (Carrillo-Moedano, 2019) y donde la sociedad se hace ella misma visible (Borja y Muxí, 2003).

Un aspecto fundamental del estado del arte sobre esta cuestión se halla en las reflexiones acerca de la representación política, de la pertenencia y de la comercialización de la vida cívica en los espacios públicos (Mitchell, 2003; Kohn, 2004; Low y Smith, 2006; Springer, 2011; Tchoukaleyska et al., 2019; Ye, 2019). Los puntos de vista son muy variados dado que, como señala Delgado (2011), el espacio público puede ser entendido como una ideología donde hay diferentes políticas y, por ello, hay un debate en la actualidad (Salcedo, 2002). No obstante, de manera general se señala la relevancia de estos espacios (Moroni y Chiodelli, 2013) porque en ellos, como demuestra Howell (1993), se desarrolla la esfera pública. Se comprueba este posicionamiento en múltiples autores. Así, Gehl (2003) defiende los espacios públicos como lugares donde se desarrolla la vida social entre los edificios y donde, por esta razón, la humanidad se humaniza. Springer (2011) los caracteriza como áreas de emancipación. Para Serrano y Stein (2019), son lugares de diálogo y transcendencia y, para Amin (2008), en los espacios públicos tiene lugar la formación de una cultura colectiva y la afirmación cívica en la vida urbana. Por el contrario, según Basson (2006), se ha reflejado una realidad idealista e ilusoria del espacio público y se han desechado visiones más oscuras, como el hecho de que hayan sido lugares de ejecución pública. Aun así, Mitchell (2003) asegura que la lucha por el espacio público es fundamental para lograr una justicia social y para el derecho a la ciudad.

La privatización del espacio público en la sociedad actual ha sido fuertemente criticada desde las geografías posmodernas de finales del siglo XX en autores como Soja (1989) o Crawford (1992). Posteriormente, ha preocupado a Kohn (2004), quien señala que las nuevas áreas residenciales urbanas son espacios privatizados. Low (2006), a través del caso de Nueva York, se refiere a la erosión del espacio público, y una de sus causas sería la privatización. En esta situación, Banerjee (2001) se cuestiona cuál será el futuro de los espacios públicos en ciudades con calles y plazas privatizadas. Németh (2009) se plantea la cuestión de la gestión del espacio público de propiedad privada, y Askin (1998) debate sobre el derecho de la libertad de expresión en los espacios pri- 
vados concluyendo que debería ser un derecho en el caso concreto y del interés principal de este artículo de los centros comerciales.

Las implicaciones que los centros comerciales suponen para la privatización del espacio público y su erosión han interesado a los académicos especializados en el estudio de estos complejos. Así, la monografía de Arcienaga et al. (2016) profundiza teóricamente en el papel de los centros comerciales en la reconfiguración de los espacios públicos y las interacciones sociales. González (2010) habla de que los centros comerciales son un simulacro del espacio público, y Calderón y García (2018) afirman que son una imitación de la vida urbana. De hecho, la literatura dominante sobre consumo y teoría urbana describe los centros comerciales como espacios cerrados que descuidan la vida urbana y la autenticidad (Simone, 2018a). Voyce (2006) responsabiliza los centros comerciales de causar una reducción del espacio público en las ciudades mediante el fortalecimiento de una ciudadanía consumista.

\subsection{Tipologias de los espacios públicos y el difícil encajamiento de los paisajes globales de los centros comerciales}

Existe una elevada heterogeneidad de espacios que se adaptan a las definiciones académicas indicadas en el apartado anterior y que pueden ser considerados como públicos. Por esta razón, es necesaria una clasificación. El trabajo de Carmona (2010), a su vez basado en estudios tipológicos previos, es válido para hallar una taxonomía de los espacios públicos. A partir de este autor, se propone una tipología de espacios públicos que se recoge en la tabla 1.

Como demuestra la tipología propuesta, establecer categorías claras de espacios públicos es complejo, sobre todo por su variedad y por las interposiciones privadas que pueden presentar. Los espacios del comercio minorista son uno de los casos más complicados, pues se encuentran en el límite entre lo público y lo privado. Como señala Monnet (1996: 11), «el comercio parece tener, en todas las civilizaciones, una función social peculiar: asegurar de la presencia de lo privado en el espacio público (...) y la de lo público en el espacio privado (lo que se podría llamar la "publicitación" de los establecimientos con "entrada libre", tiendas o plazas comerciales)». Los mercados al aire libre son un claro ejemplo (Ravetllat y Ribas, 2019), ya que en ellos hay una fuerte conexión entre lugar público, comercio y socialización desde tiempos históricos. Igualmente, si una calle, una avenida o una plaza tienen locales comerciales que remitan a un consumo colectivo, este espacio seguirá siendo un espacio público (González, 2017).

Sin embargo, la actividad mercantil minorista también se desarrolla en lugares totalmente privados. Es el caso de los centros comerciales. Por definición, un centro comercial (un mall en inglés y según su denominación original estadounidense) es un complejo promovido y gestionado por una entidad privada, es un espacio privado. Es propiedad y está operado por una sola corporación y, por tanto, está sujeto a una autoridad coercitiva y centralizada (Jackson, 1996). Se trata de un grupo de establecimientos comerciales minoristas que se planifica, desarrolla, posee y administra como una propiedad única con el 
Tabla 1. Clasificación de espacios públicos

\begin{tabular}{|c|c|c|}
\hline Tipo de espacio público & Características diferenciadoras & Ejemplo \\
\hline $\begin{array}{l}\text { Espacios urbanos naturales/ } \\
\text { seminaturales }\end{array}$ & $\begin{array}{l}\text { Espacios naturales y seminaturales den- } \\
\text { tro de las áreas urbanas bajo propiedad } \\
\text { pública. }\end{array}$ & $\begin{array}{l}\text { Bosques, montes, canales, ríos, } \\
\text { playas. }\end{array}$ \\
\hline $\begin{array}{l}\text { Espacios residuales naturales/ } \\
\text { seminaturales }\end{array}$ & $\begin{array}{l}\text { Territorios baldíos o de concentración de } \\
\text { capital improductivo que no fueron urbani- } \\
\text { zados ni preservados para uso ambiental. }\end{array}$ & $\begin{array}{l}\text { Lotes, con encerramientos de unida- } \\
\text { des prediales sin uso particular, pro- } \\
\text { piedad del estado o particulares. }\end{array}$ \\
\hline Espacios cívicos & $\begin{array}{l}\text { Las formas tradicionales de espacio } \\
\text { urbano, abierto y disponible para todos } \\
\text { y que atiende a una amplia variedad de } \\
\text { funciones. }\end{array}$ & Calles, plazas, paseos. \\
\hline Espacios públicos abiertos & $\begin{array}{l}\text { Espacios abiertos gestionados, general- } \\
\text { mente verdes y disponibles y abiertos } \\
\text { a todos, incluso si están controlados } \\
\text { temporalmente. }\end{array}$ & Parques, jardines, cementerios. \\
\hline Espacios sobrantes & $\begin{array}{l}\text { Espacios libres después del desarrollo } \\
\text { urbano, a menudo diseñados sin función. }\end{array}$ & Espacios abiertos modernistas. \\
\hline $\begin{array}{l}\text { Espacios de movimiento } \\
\text { y aparcamiento }\end{array}$ & $\begin{array}{l}\text { Espacios dominados por las necesidades } \\
\text { de desplazamiento, principalmente para } \\
\text { el transporte motorizado. }\end{array}$ & $\begin{array}{l}\text { Carreteras, autopistas, autovías, túne- } \\
\text { les, puentes, aparcamientos públicos. }\end{array}$ \\
\hline $\begin{array}{l}\text { Espacios de acceso e } \\
\text { intercambio de transportes }\end{array}$ & $\begin{array}{l}\text { Lugares de estacionamiento } \\
\text { y acceso a transportes. }\end{array}$ & $\begin{array}{l}\text { Aeropuertos, estaciones de tren, para- } \\
\text { das de autobús/tranvía, estaciones } \\
\text { de metro. }\end{array}$ \\
\hline Terceros lugares & Espacios de reunión y lugares sociales. & $\begin{array}{l}\text { Bibliotecas, centros culturales, centros } \\
\text { sociales, cafeterías y restaurantes } \\
\text { públicos. }\end{array}$ \\
\hline Espacio privado público & $\begin{array}{l}\text { Propiedad pública, pero con una especia- } \\
\text { lidad funcional o espacios determinados } \\
\text { por el usuario. }\end{array}$ & $\begin{array}{l}\text { Edificios administrativos, cuarteles, } \\
\text { comisarías, edificios educativos y de } \\
\text { sanidad, viviendas sociales. }\end{array}$ \\
\hline $\begin{array}{l}\text { Espacios para usuarios } \\
\text { seleccionados }\end{array}$ & $\begin{array}{l}\text { Espacios para grupos determinados según } \\
\text { la actividad que se realiza en ellos. }\end{array}$ & $\begin{array}{l}\text { Equipamientos deportivos (campos de } \\
\text { fútbol, gimnasios, piscinas, polideporti- } \\
\text { vos), parques infantiles. }\end{array}$ \\
\hline
\end{tabular}

Fuente: adaptación de Carmona (2010).

objetivo de maximizar las ganancias (Silva, 2018). A diferencia del bazar, del mercadillo, de la plaza de abasto, del zoco, etcétera, el centro comercial no es un lugar público ni un mercado libre (Kowinski, 2002).

Arciniega et al. (2016), basándose en una idea previa de Páramo (2011), califican los centros comerciales como sociolugares, entendidos como áreas que, aun siendo privadas, desarrollan una función socializadora. El arquitecto holandés Koolhaas (en García, 2001) va más allá y señala que el centro comercial se ha convertido en el ámbito público por excelencia. Igualmente, Silva (2018) se refiere a ellos como los nuevos «espacios (públicos)». 
En realidad, los centros comerciales son aparentemente públicos, diluyen la vida en las calles y en las plazas públicas, pero son privados y están controlados (Gehl, 2003). Son espacios públicos de gestión privada (Sarlo, 2009). Por esta razón, son más acertadas sus definiciones como espacios cuasipúblicos (Rochminska, 2017), pseudopúblicos (Cvetković y Lazarević, 2016) o semipúblicos (Augé, 1996). En definitiva, se trata de lugares de acceso público bajo control privado (Rao et al., 2017), con características de áreas públicas sin serlo.

Los centros comerciales tienen importancia para la comunidad, pero no son de propiedad pública. Son lugares privados donde el propietario está representado por personas jurídicas privadas (particulares o empresas). De hecho, generalmente son producidos por grandes corporaciones o coaliciones ad hoc de finanzas, construcción y capital comercial, con una empresa promotora especializada en su gestión, y están meticulosamente planificados (Goss, 1993).

\subsection{El paisaje global-centro comercial: áreas de consumo de éxito global y lugares de relación social}

Los centros comerciales han logrado un gran éxito económico y una gran expansión geográfica gracias a unos factores de atracción (ver tabla 2) que los han convertido en un lugar predilecto para la población. Han conseguido vincular las dinámicas de ocio y consumo que exige el mundo contemporáneo (Escudero, 2008; Arciniega et al., 2016) y en ello se encuentra la razón de su triunfo.

Los centros comerciales surgieron en Estados Unidos a mitad del siglo pasado por un modelo de vida suburbano, inspirado en unos ideales de consumo de masas y mediante la expansión del automóvil, que después será imitado en otros contextos geográficos (Simone, 2018b). Son un prototipo que se va a importar desde los Estados Unidos hibridándose con las formas comerciales minoristas domésticas en diferentes países (Simone, 2018a). Por lo tanto, se trata de una innovación estadounidense, pero que ha devenido en algo completamente global (Underhill, 2004). Hoy, se ha adoptado el centro comercial como un sitio de consumo habitual en los rincones más dispares del mundo (Sklair, 1991).

Estos complejos son paisajes globales. Suplen la función social del espacio público de las ciudades del Viejo Mundo desarrollado en Europa occidental, donde el entorno de estos lugares deja totalmente claro que el camino a la buena vida es el consumo (Sack, 1988). Además, se extienden con especial virulencia por Latinoamérica, los países del este de Europa y por la costa pacífica asiática, donde son importantes espacios de vida urbanos, eso sí, dedicados al mercado y al consumo como en los países centrales. Además, el centro comercial en los países en desarrollo y en los emergentes, con sus interiores inmaculados y recintos protegidos, deja fuera cualquier evidencia de pobreza (Sarlo, 1998) y se convierte en un halo temporal de modernidad a través del consumo (Pintaudi y Frúgoli, 1992; Simone, 2018b). Son un tipo arquitectónico global que se mueve sin esfuerzo a través de las fronteras nacionales y culturales en la corriente de la globalización neoliberal (Gosseye y Avermaete, 2019). 
Tabla 2. Recursos de los centros comerciales

\begin{tabular}{|c|c|c|}
\hline \multirow[t]{4}{*}{$\begin{array}{l}\text { Recursos } \\
\text { tangibles }\end{array}$} & Accesibilidad & $\begin{array}{l}\text { Ubicación geográfica. } \\
\text { Facilidad de acceso. } \\
\text { Aparcamiento. }\end{array}$ \\
\hline & $\begin{array}{l}\text { Mezcla } \\
\text { comercial }\end{array}$ & $\begin{array}{l}\text { Variedad de establecimientos. } \\
\text { Variedad de estilos. } \\
\text { Establecimientos singulares. } \\
\text { Establecimientos reconocidos. } \\
\text { Cadenas y franquicias. } \\
\text { Hipermercado. } \\
\text { Gran almacén. } \\
\text { Comerciantes independientes. } \\
\text { Oferta ocio y restauración. } \\
\text { Actividades de esparcimiento singulares. }\end{array}$ \\
\hline & Entorno & $\begin{array}{l}\text { Atractivo de las instalaciones. } \\
\text { Ofertas y promociones. } \\
\text { Eventos y exhibiciones. }\end{array}$ \\
\hline & Tecnología & $\begin{array}{l}\text { Tecnologías de información y comunicación (tic) para la gestión. } \\
\text { Tic para interactuar oferta y demanda. }\end{array}$ \\
\hline \multirow[t]{5}{*}{$\begin{array}{l}\text { Recursos } \\
\text { intangibles }\end{array}$} & Servicios & $\begin{array}{l}\text { Aparcamiento gratuito. } \\
\text { Atención al cliente. } \\
\text { Amplitud del horario de apertura. } \\
\text { Servicios complementarios. }\end{array}$ \\
\hline & Personal & $\begin{array}{l}\text { Profesionalidad. } \\
\text { Formación. } \\
\text { Motivación. } \\
\text { Especialización. }\end{array}$ \\
\hline & Financieros & $\begin{array}{l}\text { Formas de pago. } \\
\text { Aplazamiento de pago. } \\
\text { Financiación. }\end{array}$ \\
\hline & Estrategia & $\begin{array}{l}\text { Satisfacción y fidelización clientela. } \\
\text { Campañas publicitarias. } \\
\text { Estudios de mercado. } \\
\text { Relaciones institucionales. } \\
\text { Análisis de la competencia. } \\
\text { Actividades de marketing. }\end{array}$ \\
\hline & $\begin{array}{l}\text { Gestión } \\
\text { comercial }\end{array}$ & $\begin{array}{l}\text { Coordinación de intereses de gestión y comerciales. } \\
\text { Administraciones de locales vacíos. } \\
\text { Gestión económico-financiera. }\end{array}$ \\
\hline
\end{tabular}

Fuente: Marín y Martín (2013).

A escala global, las compras se han convertido en actividades recreativas y el centro comercial en un refugio (Cvetković y Lazarević, 2016). El mall es la "catedral del consumo" (Moulian, 1999). En su interior, el comportamiento y las interacciones se definen por el propósito principal de este espacio: el 
consumo (Cvetković y Lazarević, 2016). Los centros comerciales crean un facsímil de la ciudad tradicional con sus calles, mercados y plazas (Rao et al., 2017). Las tiendas, los escaparates, las luces altas, las fuentes, el mobiliario urbano y los jardines interiores representan un intento de reproducir las calles comerciales de la ciudad tradicional compacta dentro de un espacio privado cerrado (Gharbi y Knight, 2017). Así, por ejemplo, se recurre a la presencia de la naturaleza, aunque domesticada en un jardín, una recreación ficticia de un espacio natural ${ }^{2}$, para mitigar la alienación inherente a la producción y el consumo de mercancías (Goss, 1993), pero, en realidad, son espacios para el consumo (Shields, 1992). La iluminación, la música ambiente y hasta los olores se diseñan para crear la mejor sensación posible y abrir el deseo de comprar. Utilizan varias formas de disciplina espacial, con una planificación paternalista para guiar al visitante hacia la compra (Thaler y Sunstein, 2003). El objetivo es lograr un mayor consumo por parte de los visitantes y una mayor ganancia para los dueños del lugar (López, 1999; Gosseye y Avermaete, 2019).

Sklair (2010) habla del centro comercial como un lugar instrumentable en el que pocos problemas se dejan al azar, ya que el espacio está cuidadosamente diseñado en detalle y monitoreado y modificado cada vez que la utilidad percibida del consumidor es subóptima. La arquitectura, el diseño, la ornamentación y la gestión cotidiana se basan en una forma de vida consumista (Styhre, 2019). Crean espacios hiperreales, «más reales que la realidad», dirigidos al consumo y sustituyendo a «lo verdadero» (Escudero, 2008), con ambientes artificiales (López, 1999). Son recintos aislados frente al caos y la decadencia urbana exteriores y frente a las inclemencias del tiempo. En ellos, todo funciona: aire acondicionado, música de fondo, seguridad, etcétera (Gorelik y Silvestri, 1991). Medina (1998) los calificó metafórica y visualmente como «burbujas de cristal», y Styhre (2019) afirma que crean perfectos «mundos-burbuja». Las áreas traseras (backstages) están ocultas por paisajismo, paneles pintados y construcción subterránea para proteger a los clientes del conocimiento de las actividades que tienen lugar allí, para preservar así el mito del producto puro y abstracto para la venta. «El acceso a estas áreas es imposible para aquellos que no conocen el plan» (Goss, 1993: 93).

Además, en la actualidad y cada vez más, en los centros comerciales hay un conjunto de instalaciones de ocio mercantilizado y de actividades culturales y recreativas con un objetivo claro: crear espacios amigables que inviten a quedarse y consumir (Escudero, 2008; Silva, 2018). Han ido agregando elementos que favorecían el ocio (González, 2017). Este hecho ha dado lugar a que tengan un carácter cada vez más hedonista (Iso, 2009) y a que sustituyan algunas funciones no utilitarias ejercidas por los centros urbanos tales como el ocio y la sociabilidad.

2. El centro comercial Jewel, abierto en 2019 en el aeropuerto de Singapur, lleva la imitación de la naturaleza al extremo recreando un bosque tropical y con la construcción de la cascada artificial de interior más elevada del mundo. El lector puede comprobarlo en este vídeo de los autores: <https://www.dropbox.com/s/ivovwuzpw8sex2u/Jewel\%20\%28Singapur\%29. MOV? $\mathrm{dl}=0>$. 
Figura 1. Diferentes personas usan el Raffles City Shopping Centre en Singapur para pasear, charlar, descansar y consultar su teléfono móvil

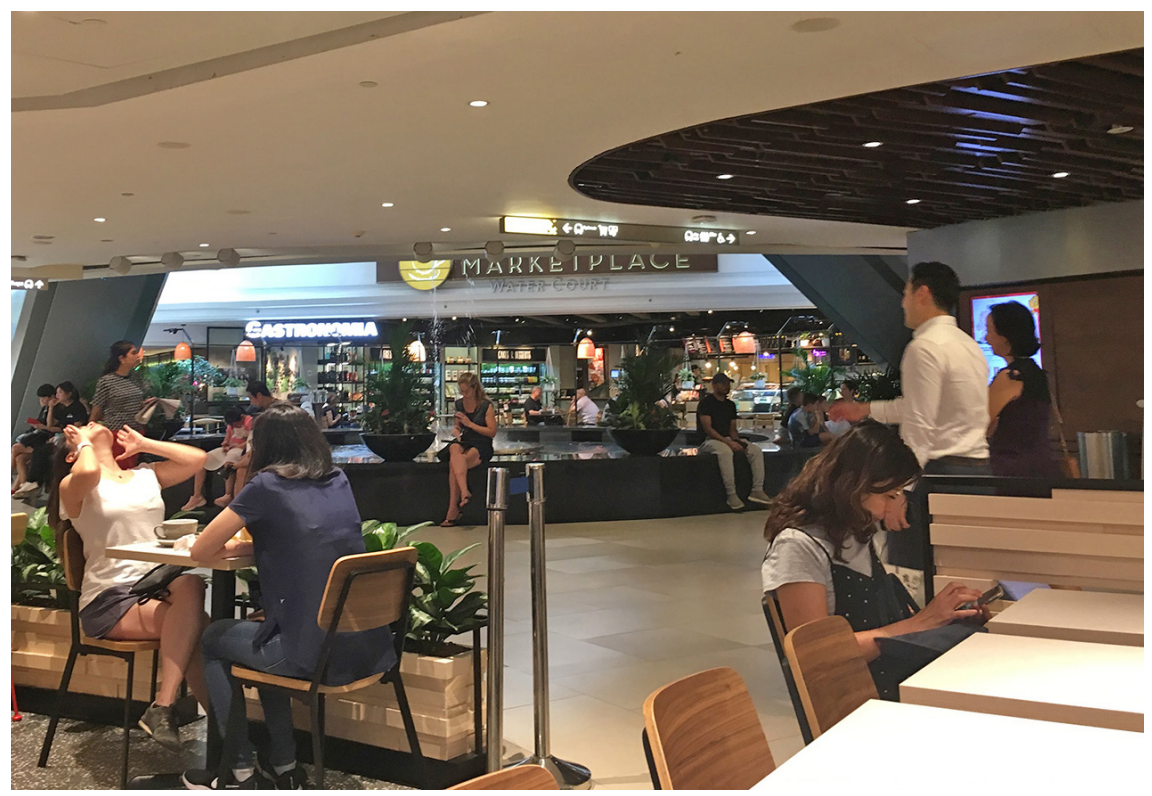

Fuente: fotografía de L. A. Escudero, 13 de agosto de 2019.

Estas instalaciones se han convertido en espacios para caminar, pasear, deambular, saludar a viejos conocidos, encontrarse con amigos, charlar, sentarse unos minutos, tomar café o ver quién pasa (López, 1999), por supuesto, comprando. El flâneur ${ }^{3}$ del centro comercial no puede escapar al imperativo del consumo (Goss, 1993). Salcedo (2003) demuestra que las personas no consideran que estos complejos sean simplemente lugares comerciales, sino que, a menudo, los perciben como espacios en los que se satisfacen algunas necesidades psicológicas, como la interacción social o como la relajación y el ocio (ver figura 1).

Es evidente que los centros comerciales se han convertido en puntos principales de relación social para sectores cada vez más amplios de la población (Crawford, 1992; Parlette y Cowen, 2011). En particular, destacan como espacios de consumo, de reunión y de sociabilización para determinados grupos sociodemográficos como son la población femenina (Michon et al., 2008; Jackson et al., 2011), la juvenil (Anthony, 1985; Bermúdez, 2008; Jiménez et al., 2009) y las familias con niños (Timothy, 2005; Ali y

3. Aquel paseante que deambulaba por la ciudad sin ningún propósito en especial más que contemplar la vida y que describían figuras notables del siglo XIX como Baudelaire o Poe y admiraba Benjamin en la primera mitad del Xx (Tester, 1994). 
Anuar, 2013), así como en algunos países, como España, la población jubilada (Escudero, 2008).

En definitiva, al no desarrollarse solo prácticas de consumo, los centros comerciales acaban siendo constructores efectivos de identidad, de memoria colectiva y de nuevos hábitos de vida cotidiana. Son receptáculos de estilos de vida y de procesos de socialización gradualmente complejos (Silva, 2018). Thrift (2008) señala que acaban creando incluso respuestas afectivas. En este sentido, no son realmente «no lugares» como los denominó Augé (2012), sino más bien son "pseudolugares» (Goss, 1993). Se convierten en sitios con una significación simbólica en la vida cotidiana (Simone, 2018a), que superan ampliamente su rol original, la venta minorista. En el espacio de los centros comerciales se producen encuentros sociales (Miller y Laketa, 2018).

Sin embargo, los centros comerciales son lugares delimitados que niegan la autenticidad de la vida urbana. Desde el punto de vista físico y psicológico, el aislamiento segrega los centros comerciales de la realidad urbana (López, 1999). Se pierde la relación vecinal propia del auténtico espacio público a cambio del deambular entre la compra y el ocio. Aunque haya contacto social, lo primordial no es el vínculo entre personas, sino apropiarse de las ofertas del centro comercial. En lugar de permitir contactos, fomenta un cierto tipo de anonimato descuidado (Álvarez y Villarejo, 2003). Es en los espacios públicos de las ciudades donde realmente se puede producir el trato más alto en las interrelaciones humanas (Nahavandi et al., 2017). Peñalosa (2017: 7) afirma que «en una ciudad sana los espacios públicos, y no los centros comerciales, son el lugar de encuentro».

Estos paisajes globales se apropian de funciones sociales y son lugares de entretenimiento y ocio, encuentran vida en las dinámicas socioculturales derivadas de su uso (Arcienaga et al., 2016), pero nunca llegaron a cumplir realmente la función cívica, cultural y social que su inventor, el arquitecto Victor Gruen, pensó para ellos (Hardwick, 2004). Gruen fue el autor del Southdale Center en 1956, en Edina (Minesota), localidad del área metropolitana de Mineápolis, el primer centro comercial cerrado de los Estados Unidos, así como de otros muchos por todo el país. Southdale Center fue un arquetipo que se reprodujo casi fielmente, salvo pequeñas modificaciones locales, en miles de lugares en Norteamérica y, posteriormente, en el resto del mundo (Gladwell, 2004). El arquitecto coetáneo Frank Lloyd Wright fue uno de los primeros críticos que advirtió de los peligros del centro comercial y señaló que Southdale tenía todos los males de la calle pública y nada de su encanto (Bures, 2014). Sin embargo, Gruen pensó estos espacios como nuevos centros urbanos de la creciente periurbanización de las ciudades estadounidenses, una alternativa para las que consideraba decadentes áreas centrales (Gruen y Smith, 1960). Pero el invento de Gruen fue apropiado por desarrolladores con mentalidad de negocio (Styhre, 2019), que se centraron en la visión mercantil y capitalista del mall (ver figura 2). 
Figura 2. Southdale Center, el primer centro comercial cerrado del mundo, hoy un típico mall periurbano

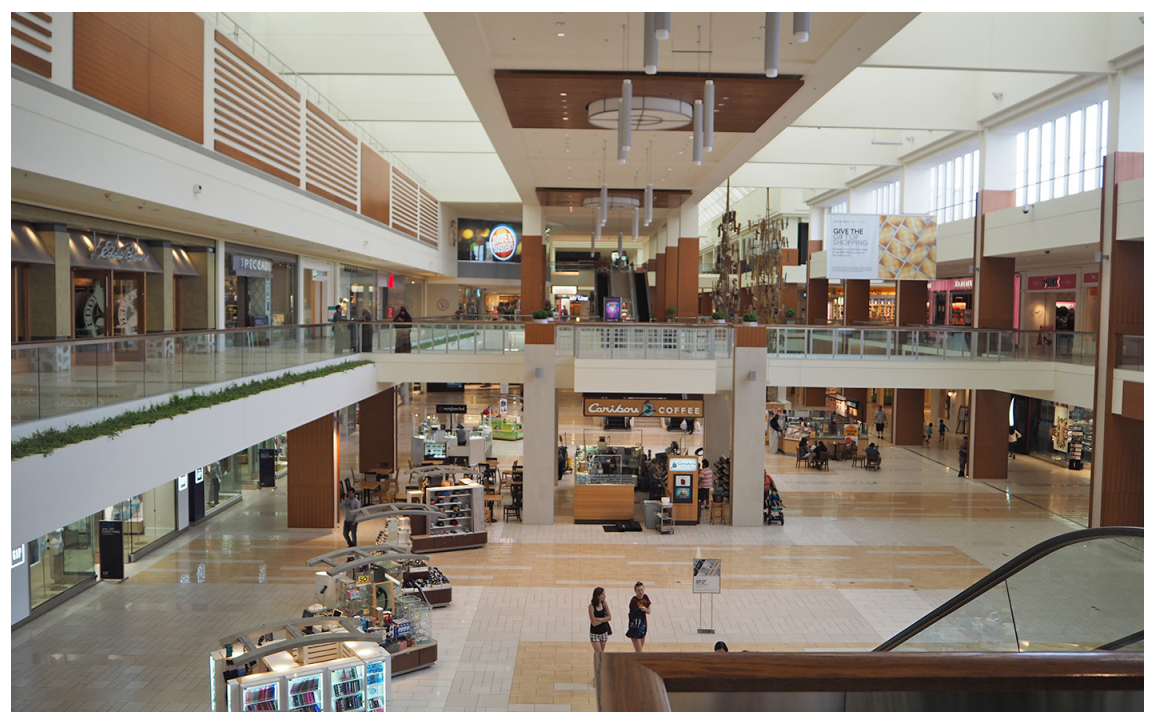

Fuente: fotografía de L. A. Escudero, 5 de agosto de 2018.

\subsection{Los centros comerciales como sustitutos de los espacios urbanos públicos, calles y plazas, por un urbanismo comercial y privado}

Estrictamente, la creación de un centro comercial no implica automáticamente ninguna privatización de un espacio público, por el contrario, comporta nuevos lugares colectivos para la comunidad (Chiodelli y Moroni, 2015). Normalmente se ubican en suelo vacío de propiedad privada que es transformado en un área abierta a todos los clientes del centro comercial. En realidad, el proceso de privatización no es directo, sino mediante tres posibilidades distintas de reconfiguración de los espacios público-privados: ocupan terrenos libres dentro de la ciudad, sustituyen los usos públicos de calles y plazas y se erigen en los centros de las áreas periurbanas.

Las parcelas de suelo donde se construyen los centros comerciales son lugares que permanecían vacíos en la ciudad y que pasan a ser ocupados para un uso privado en vez de ser empleados para alguna utilidad pública. Invaden grandes vacíos urbanos que podrían ser utilizados social y públicamente. Este es su primer y más directo modo de reconfiguración de los espacios público-privados en las ciudades. Esto se demuestra a través de dos casos distintos.

El primero es el del centro comercial La Vaguada, en Madrid, abierto en 1983 y que fue estudiado por el sociólogo Castells (en Susser, 2001). La idea de construir un centro comercial en el terreno del barrio madrileño del Pilar donde hoy se sitúa, en un área abierta rodeada por torres de viviendas, provocó el rechazo de los vecinos en los años setenta. Estos reivindicaban 
la utilización de la parcela vacía para mejorar los equipamientos públicos del barrio y crear ahí zonas verdes y de recreo. Solicitaban, en definitiva, un espacio público dentro de un barrio residencial muy densamente poblado y con carencia casi total de áreas libres y parques. Por este motivo, los residentes se movilizaron contra la construcción de un gran centro comercial en medio del Pilar. También se rechazaba el proyecto aduciendo que incrementaría la congestión del tráfico y arruinaría a los pequeños comerciantes. Sin embargo, las presiones de la empresa promotora fueron más poderosas que las de los vecinos y el centro comercial se construyó. La Vaguada nunca llegó a ser «un espacio libre para vecinos liberados» (Castells en Susser, 2001: 216). No obstante, hoy el centro comercial es el área habitual de compra del barrio, así como un lugar donde pasear, tomar un café o pasar el rato, como se observó reiteradamente a través de trabajo de campo y en conversaciones informales con sus usuarios. Por ejemplo, personas jubiladas confesaban que acudían regularmente simplemente para caminar, sentarse y hablar con sus vecinos y amigos, que también iban al centro con estos fines de socialización.

Un segundo caso se presenta en el sur de la ciudad de Bogotá, en la década de los ochenta, donde se vería la emergencia de un centro comercial ensamblado a la ciudadela de edificios de cinco plantas como viviendas de interés social para las clases populares: el centro comercial Ciudad Tunal. Como otras unidades de su tipo, estaba pensado para constituirse en prótesis de la autosuficiencia de todo un sector de la ciudad. Sin embargo, a diferencia de otros centros ubicados en barrios urbanizados de clase media y alta ${ }^{4}$, este abarca una vecindad directa con barrios populares caracterizados por la autoconstrucción, práctica propia en la urbanización colombiana de la década de los sesenta, y el comercio abierto o de calle como elementos naturales de su paisaje urbano. Tal contigüidad implicó otra clase de encuentros con el cambio espacial y una menor dependencia de la ciudadela con relación al centro comercial como prótesis, en la medida en que los barrios junto a la urbanización proveían de todos los productos, desde los alimentarios hasta los de tipo suntuario. Así pues, el acoplamiento del centro comercial se realizaría en articulación con otras instituciones que tuvieron su habitáculo allí, como fueron la iglesia, el centro de salud y la biblioteca — no precisamente el comercio-, con los cuales el centro comercial terminaría desarrollando sinergias con las que se configuró la territorialidad de la marca comercial Ciudad Tunal (ver figura 3).

Entre los propósitos del proyecto que se establecen como consignas de la instalación territorial, se propone evitar la monotonía que «les permite a sus residentes crear su propia identidad y configuración social» $(\mathrm{BCH}, 1988$ : 14). De tal manera que, en la disposición de la marca territorial de Ciudad

4. Un interesante ejemplo localizado en otro ámbito de Latinoamérica es el del centro comercial de La Florida, en Santiago de Chile, estudiado por Flores (2019). Esta autora demuestra cómo este mall es el espacio público, privado de uso, más exitoso en la vecindad de clase media del mismo nombre. Por contraste, las calles, parques y plazas del barrio están deficientemente mantenidas y carecen de vida pública. 
Figura 3. Centro comercial Ciudad Tunal (Bogotá), con la entrada a la iglesia subterránea en la imagen de la derecha

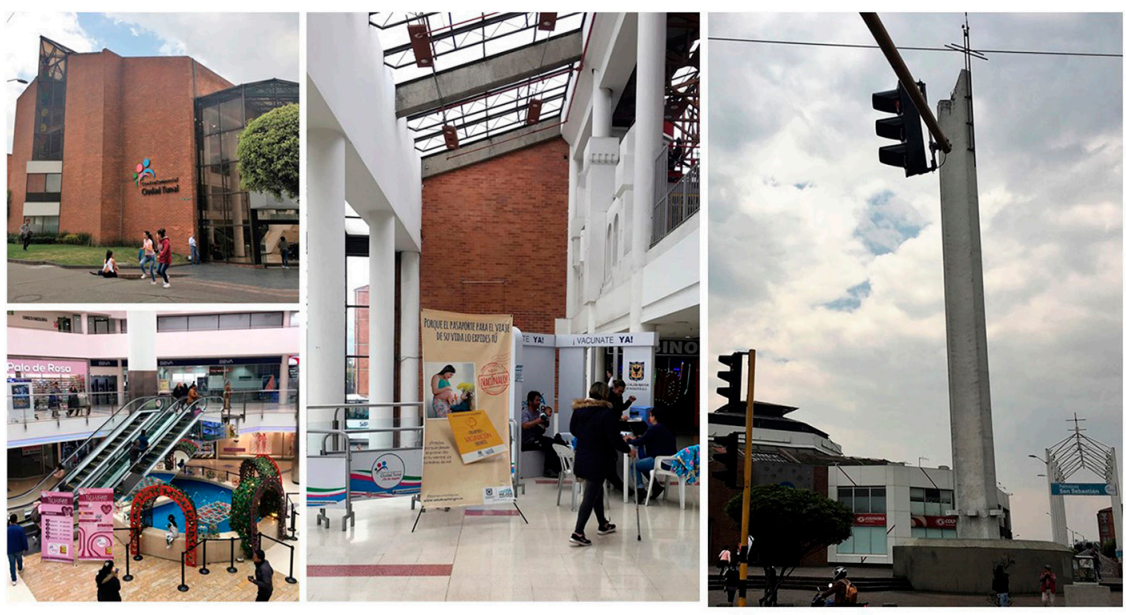

Fuente: fotografías de L. A. Escudero, 17 de septiembre de 2019.

Tunal, su centro comercial, del mismo nombre, se constituye como agente geográfico-semiótico de cohesión social a partir de su pertenencia al modelo de la ciudadela y a sus propósitos misionales. Así, una de las características del diseño del centro comercial es su ubicación, en la cual integra la plaza pública como lugar social de reunión y la iglesia subterránea como vecindad institucional. A diferencia de otros centros comerciales de Bogotá que integraron el ritual católico a través de las eucaristías dominicales y servicios religiosos en Semana Santa exclusivamente, el Tunal está arquitectónicamente ligado a la iglesia (ver figura 3), que, aunque no fue la idea primigenia - pues el lugar que ocupa estaba diseñado como casino subterráneo-, se consolida como adaptación del centro como prótesis comercial a la institucional religiosa, en este caso dando continuidad al modelo tradicional del municipio colombiano o tecnología parroquial como lo propone Zuleta (2013).

Los dos casos estudiados evidencian que, como señalan Garzón et al. (2020), los centros comerciales son transformaciones en el territorio y en la vida cotidiana. En los barrios residenciales madrileño y bogotano donde se localizan, sus vecinos los han adoptado en su actividad diaria y constituyen, con sus formas globales, parte de su paisaje urbano. Especialmente significativo es el caso de las eucaristías religiosas en Ciudad Tunal, donde, como también ha constatado Molina (2019), se observó cómo se pasaba de la experiencia religiosa - la misa en el complejo- a la del consumo. Los feligreses se convertían en clientes del centro comercial. Los dos casos locales analizados demuestran, como defienden Arciniega et al. (2016), que unos espacios de venta minorista han sobrepasado esta función para asumir el rol habitual de los espacios públicos. Así, las personas que van a estos centros comerciales los utilizan, como se 
señaló, para pasar su tiempo libre, pasear, deambular, hacer relaciones sociales, etcétera, al mismo tiempo que abandonan o emplean menos las calles y plazas de los dos barrios estudiados. Este proceso es observado también por Cvetković y Lazarević (2016) en los centros comerciales de Belgrado, dentro del carácter global de estos espacios.

La Vaguada y Ciudad Tunal son centros comerciales que se han convertido en espacios multifuncionales que sustituyen a otros lugares de la ciudad. Estos dos casos locales concretos observados suponen una confirmación de trabajos académicos previos que resaltaban este hecho (López Levi, 1999; Lulle y Paquette, 2006; Escudero, 2008; González, 2010; Chiodelli y Moroni, 2015). Frente a Bruegmann (2005), defensor del modelo de la ciudad difusa — que señala que las críticas a la suburbanización y a los centros comerciales son exageradas y que realmente son espacios públicos con una importante labor social—, se considera necesario, a partir de las experiencias de los centros comerciales analizados, una reflexión de la pérdida actual de la esfera pública por áreas particulares de consumo con reglas privadas.

Tras estudiar estos dos casos, de manera general se puede deducir que los visitantes de los centros comerciales los usan como si fuesen un espacio público sin serlo y, obviamente, a cambio dejan de utilizar las áreas realmente públicas. De este modo, actividades y funciones previamente reservadas a los espacios urbanos públicos tienen lugar ahora en su interior (ver figura 4). El centro comercial es el reverso de la cultura ciudadana, la negación del espacio público donde se mezclaba el trabajo y el descanso. En su lugar reina ahora el medio privado, controlado por las medidas de seguridad privadas y polarizado por el negocio (Verdú, 1996). Las plazas (Allen, 2006; Göbel, 2016) y las calles (Jacobs, 1993) son propiedad del actor público, expresamente pagadas

Figura 4. Fuente de los deseos en la Lonja de Valencia y en el Mall of America (Mineápolis)

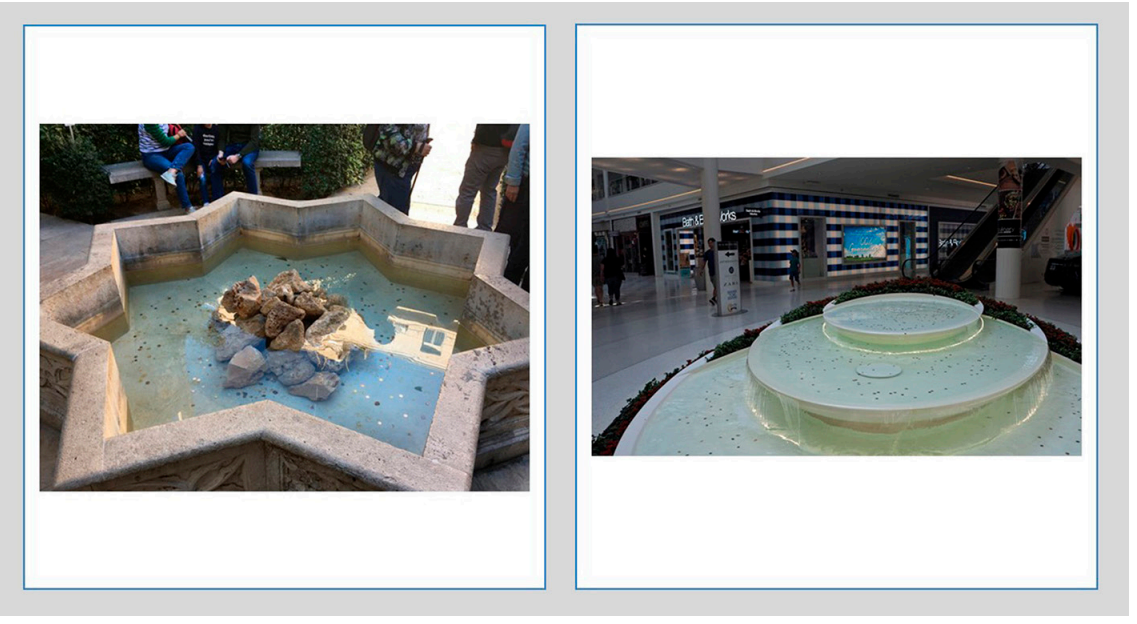

Fuente: fotografías de L. A. Escudero, 26 de octubre de 2019 y 9 de agosto de 2018. 
por los contribuyentes, mantenidas y administradas por empleados públicos, formal y específicamente dedicadas al uso público. Por el contrario, el centro comercial privado no es ninguna de estas cosas (Chiodelli y Moroni, 2015). Es administrado por un actor privado involucrado en una empresa comercial $\mathrm{y}$ depende de sus clientes para sobrevivir.

Finol (2006) destaca que mientras que la plaza pública viene definida por una estructura radial, del centro hacia la periferia, el centro comercial es concéntrico, dirigido hacia un círculo interior de tiendas. En el caso de las calles comerciales tradicionales de las ciudades, estas invitan al azar, al devaneo, al encuentro y al paseo, y no responden a técnicas conductistas para atraer al potencial cliente a una tienda o servicio. Las calles y plazas públicas son espacios públicos que se caracterizan por la multifuncionalidad, por el intercambio y por los lugares de encuentro y de expresión (Borja y Muxí, 2003). Los centros comerciales reproducen sus ambientes urbanos: vías y señalización, bancos, fuentes, etcétera (Lulle y Paquette, 2006), pero las calles de los centros comerciales nos dirigen a la compra, al cine, a la cafetería...

El espacio público del centro comercial, sus calles y plazas, no deja de ser, paradójicamente, un espacio privado delimitado. Por ejemplo, las plazas y calles, por lo general, no tienen horarios, los centros comerciales sí y su actividad va a estar limitada a los mismos. Estos paisajes globales forman islas urbanas separadas. Crean enclaves aislados y cerrados (o parcialmente cerrados) dentro de una urbe, lo que da lugar a una "ciudad dentro de una ciudad" " para crear una ciudad «perfecta» (Rochminska, 2017).

\section{Conclusiones}

La conclusión principal obtenida en este trabajo es que los centros comerciales, como paisajes globales, están reconfigurando los espacios públicos a escala planetaria mediante una apropiación de sus funciones. No son áreas colectivas ni comunitarias, sino recintos privados, con un control, una gestión y una regulación propios. Su diseño se rige por técnicas conductistas que inducen al consumo, y los eventos socioculturales que programan también buscan un fin último económico: la obtención de beneficios por la venta minorista de bienes y servicios.

Estos paisajes globales han logrado un gran éxito económico y una gran expansión geográfica gracias a sus múltiples recursos tangibles (accesibilidad, entorno, mezcla comercial y tecnología) e intangibles (estrategia, financieros, gestión comercial, personal y servicios), que los han convertido en un lugar de gran atractivo para los ciudadanos y a escala planetaria. Como paisajes de la globalización, su función es proporcionar la cultura global de consumo de la sociedad

5. El centro Marineda City, en La Coruña (España), da la bienvenida a sus visitantes mediante la frase "La ciudad es tuya», como el lector puede ver en la imagen de los autores disponible en: <https://www.dropbox.com/s/6wwmih25a2gha0p/Marineda $\% 20$ City $\% 20 \% 28 \mathrm{~A} \% 20$ Coru\%C3\%B1a\%29.JPG?dl=0>. 
actual. Al mismo tiempo, los centros comerciales se han convertido en espacios para múltiples actividades sociales (pasear, encontrarse con amigos, charlar, descansar, etcétera) y no son solo lugares comerciales. Por ello, son constructores de identidad, de memoria colectiva y de nuevos hábitos de vida cotidiana con procesos de socialización complejos.

Los centros comerciales se apropian de funciones sociales y, al hacerlo, reemplazan los espacios públicos donde se desarrollaban anteriormente. Reconfiguran los espacios público-privados mediante una sustitución de los usos públicos de las calles y las plazas. En los dos casos estudiados de La Vaguada en Madrid y Ciudad Tunal en Bogotá, localizados en espacios residenciales donde la comunidad vecinal solicitaba otros usos de carácter público, se han terminado desarrollando sinergias para configurar una nueva territorialidad y un fuerte uso social que reemplazan los lugares públicos. Mediante un proceso de sustitución, se han convertido en áreas de encuentro, pero los centros comerciales no son ni las calles ni las plazas. Son el reverso de la cultura ciudadana, la negación del espacio público por un medio privado absolutamente controlado y polarizado por el lucro y el negocio de los comercios y empresas ubicados en su interior, de los propietarios de los centros comerciales y de sus promotores. Tras el análisis conceptual y el trabajo de campo realizados, se responde afirmativamente a la pregunta de investigación: los centros comerciales son una apropiación privada de los espacios públicos urbanos a escala global. Con ello, esta investigación supone una aportación teórica y práctica que respalda teorías académicas anteriores y que desmonta la opinión de Bruegmann (2005), quien señala que las críticas a los centros comerciales son exageradas y que realmente son espacios públicos. Sin embargo, no son los centros comerciales en sí, sino la sustitución que hacen de las áreas comunes de uso ciudadano lo que se critica en el artículo. Como propone Mitchell (2003), se defiende una lucha por el espacio público.

Este trabajo conceptual y teórico pretende ser un marco de referencia y una aportación al estado del arte de los temas analizados. No obstante, y al igual que cualquier investigación social, presenta limitaciones. $\mathrm{Al}$ abordar de forma teórica un proceso global, las desigualdades y los procesos locales no han sido correctamente tratados. No obstante, se considera que Salcedo (2003) y Gosseye y Avermaete (2019) ya han analizado ampliamente estos aspectos. Además, el artículo abre un campo de investigación para otros estudios empíricos o teóricos que profundicen en las diferencias dentro de estos paisajes globales a partir de sus ideas teóricas generales. También, por motivos de extensión máxima del texto, no se han podido tratar de forma extensa interesantes aspectos de la reconfiguración de los espacios públicos por los centros comerciales como el de la seguridad o el de su carácter apolítico. Es el propósito de los autores poder profundizar en estas temáticas en otros trabajos posteriores.

Finalmente, el presente artículo tiene un objetivo teórico y estructural, pero los centros comerciales conocen importantes dinámicas coyunturales que deben ser objeto de investigación. Las evoluciones de decadencia y cierre, principalmente en su lugar de origen, los Estados Unidos, y los efectos de la pandemia 
de la COVID-19 en los centros comerciales son cuestiones que se deben seguir investigando en el futuro, en el primer caso, o que deben ser observadas y analizadas en función de la evolución de la crisis sanitaria presente, en el segundo.

\section{Referencias bibliográficas}

Ali, Mohd y ANUAR, Khairui (2013). «A structural equation modeling approaches on factors of shopping mall attractiveness that influence consumer decision-making in choosing a shopping mall». Journal of Global Business Economy, 6 (1), 63-76.

ALLEN, John (2006). «Ambient power: Berlin's Potsdamer Platz and the seductive logic of public spaces». Urban Studies, 43 (2), 441-455. $<$ https://doi.org/10.1080/00420980500416982>

Álvarez Cantalapiedra, Santiago y Villarejo Galende, Helena (2003). «La regulación de los grandes centros comerciales: una aproximación sociológica y jurídica». Revista Derecho, 15, 131-155.

AmIN, Ash (2008). «Collective culture and urban public space». City, 12 (1), 5-24. $<$ https://doi.org/10.1080/13604810801933495>

ANTHONY, Kathryn H. (1985). «The shopping mall: A teenage hangout». Adolescence, 20, 307-312.

Arciniega Solano, Elena; Guerrero Sierra, Hugo F. y Wilches TinjacÁ, Jaime A. (2016). Y ahora... ¿ ¿ dónde vamos? Los centros comerciales en la reconfiguración de los espacios públicos y las interacciones sociales. Bogotá: Grupo Editorial Ibánez.

AsKIN, Frank (1998). «Free speech, private space, and the constitution». Rutgers Law Journal, 29 (3), 947-961.

AUGÉ, Marc (1996). Los no-lugares. Espacios del anonimato. Una antropología de la sobremodernidad. Barcelona: Gedisa.

Banco Central Hipotecario (BCH) (1988). El Tunal. Proyecto de conjunto para una ciudad dentro de la ciudad. Bogotá: $\mathrm{BCH}$.

BANERJEE, Tridib (2001). «The future of public space: Beyond invented streets and reinvented places». Journal of the American Planning Association, 67 (1), 9-24. $<$ https://doi.org/10.1080/01944360108976352>

BAricco, Alessandro (1996). Seda (X. González y C. Gumpert, trads.). Barcelona: Salvat, 2001.

BASSON, Steve (2006). «"Oh comrade, what times those were!". History, capital punishment and the urban public square». Urban Studies, 43 (7), 1.147-1.158. <https://doi.org/10.1080/00420980600711704>

BAUmAN, Zygmunt (2000). Modernidad líquida (M. Rosenberg y J. Arrambique, trads.). Ciudad de México: Fondo de Cultura Económica, 2003.

BENACH, Núria y AlBET, Abel (2019). «David Harvey: diálogo abierto entre la abstracción teórica y la geografía de la urbanización». En: BENACH, Núria y AlBET, Abel (eds.). David Harvey. La lógica geográfica del capitalismo. Barcelona: Icaria, 301-336.

BERMÚDEZ, Emilia (2008). «Malls: territorios y objetos de consumo simbólico en la construcción de representaciones de identidades juveniles: un estudio cualitativo sobre algunos grupos de jóvenes en la ciudad de Maracaibo, Venezuela». Revista Argentina de Sociología, 6 (11), 96-120.

BerQUE, Augustin (1990). Mediance. De milieux en paysages. Montpellier: GIP-Reclus. - (2009). El pensamiento paisajero. Madrid: Biblioteca Nueva.

BORJA, Jordi y MuXí, Aida (2003). El espacio público: ciudad y ciudadanía. Barcelona: Electa. 
Bruegmann, Robert (2005). Sprawl. A compact history. Chicago: The University of Chicago Press.

BurES, Frank (2014, 25 de julio). «The life and death of malls». Minnpost. Recuperado de <https://www.minnpost.com/thirty-two-magazine/2014/07/life-and-deathmalls $>$ [consulta: 5 de septiembre de 2020].

Calderón Calderón, Basilio y García Cuesta, José Luis (2018). «La estructura de las ciudades españolas: un complejo entramado de relaciones entre permanencias y cambios, formas y usos». Boletín de la Asociación de Geógrafos Españoles, 77, 283-313. <https://doi.org/10.21138/bage.2542>

CARmona, Matthew (2010). "Contemporary public space, part two: Classification». Journal of Urban Design, 15 (2), 157-173. <https://doi.org/10.1080/13574801003638111>

Carmona, Matthew; Heath, Tim; Oc, Taner y Tiesdell, Steve (2012). Public Places, Urban Spaces. Londres: Taylor \& Francis.

Carr, Stephen; Francis, Mark; Rivlin, Leanne G. y Stone, Andrew M. (1992). Public space. Nueva York: Cambridge University Press.

Carrillo-Moedano, Enid A. (2019). «Miradas a la ciudad: el estado del conocimiento en los estudios del espacio público urbano». Uno Sapiens, 3, 5-11.

Castells, Manuel (1971). Problemas de investigación en sociología urbana. Madrid: Siglo XXI.

Castiblanco, Andrés (2015). «Elementos críticos en la transición tecnológica de la experiencia y el relato urbano». En: Piedrahita Echandía, Claudia; Díaz Gómez, Álvaro y Vommaro, Pablo (eds.). Pensamientos críticos contemporáneos. Análisis desde Latinoamérica. Bogotá: CLACSO-Universidad Distrital, 217-238.

- (2018). Marcas y marcajes. Otras memorias y luchas en Bogotá a finales del siglo XX y principios del XXI. Bogotá: Universidad Distrital Francisco José de Caldas.

Chiodelli, Francesco y Moroni, Stefano (2015). "Do malls contribute to the privatization of public space and the erosion of the public sphere? Reconsidering the role of shopping centres». City, Culture and Society, 6, 35-42. <https://doi.org/10.1016/j.ccs.2014.12.002>

Cobley, Paul (2017). "Semiótica, observación y las "ciencias del saber"». En: PARDO ABril, Neyla G. (ed.). Semióticas. Materialidades, discursividades y culturas. Bogotá: FELS-Universidad Nacional de Colombia-Instituto Caro y Cuervo, 34-52.

CONSEJO DE Europa (2000). «Convenio Europeo del Paisaje». Recuperado de <https:// rm.coe.int/CoERMPublicCommonSearchServices/DisplayDCTMContent?docu mentId $=09000016802 \mathrm{f3fbd}>$ [consulta: 5 de septiembre de 2020].

Cosgrove, Denis (2008). Geography \& Vision. Seeing, Imagining and Representing the World. Nueva York: I. B. Tauris.

CRAWFORD, Margaret (1992). «The world in a shopping mall». En: SORKIN, Michael (ed.). Variations on a Theme Park: The New American City and the End of Public Space. Nueva York: Hill and Wang, 3-30.

Cvetković, Marija y Lazarević, Eva V. (2016). «The image of the city vs. semipublic of shopping malls: Case study of Belgrade». En: 3rd International Academic Conference on Places and Technologies. Recuperado de <https://www.academia. edu/attachments/58955197/download_file?st=MTU2Njk5MDY4OCw3OS4x MDkuMTc4LjEyMyw0NTIxNDQ1Mg\%3D\%3D\&s=profile> [consulta: 5 de septiembre de 2020].

Daniels, Stephen; Delyser, Dydia; Entrikin, Nicholas y Richardson, Douglas (2011). «Introduction». En: Daniels, Stephen; Delyser, Dydia; Entrikin, 
Nicholas y RichardSON, Douglas (eds.). Envisioning Landscapes, Making Worlds. Geography and the Humanities. Nueva York: Routledge, XXVII-XXXII.

Delgado, Manuel (2011). El espacio público como ideología. Madrid: Catarata.

ESCUDERO GÓMEZ, Luis A. (2008). Los centros comerciales, espacios postmodernos de ocio y consumo: un estudio geográfico. Cuenca: Universidad de Castilla-La Mancha.

EsTÉVEZ VILLARINO, Brais (2012). "La idea de espacio público en geografía humana. Hacia una conceptualización (crítica) contemporánea». Documents d’Anàlisi Geogràfica, 58 (1), 137-163.

FINOL, José Enrique (2006). «Globalización, espacio y ritualización: de la plaza pública al mall». Espacio Abierto, 14 (4), 573-588.

Flores CASTILlo, Mónica A. (2019). «The mall as a public space: The case of La Florida, Santiago». SIUU XI. Seminario Internacional de Investigación en Urbanismo. <https://doi.org/10.5821/siiu.6702>

GARCÍA GARCÍA, Antonio (2011). «El valor de la perspectiva geográfica para el análisis de los espacios públicos urbanos». Boletín de la Asociación de Geógrafos Españoles, 55, 281-301.

García Herrera, A. (2001, 17 de febrero). «Mutaciones». El Pais, 18.

García VÁZQUEZ, Carlos (2004). Ciudad hojaldre. Visiones urbanas del siglo XXI. Barcelona: Gustavo Gili.

Garzón Medina, Carolina; Sánchez Arismendi, Aidaluz; Neira, M. Paula; Cañón, Angélica; PÁez, Alejandra; Mendoza, Angie y GonzÁlez, Jennifer (2020). "Centros comerciales: transformaciones en el territorio y en la vida cotidiana». Universidad Santo Tomás. Recuperado de <https://repository.usta.edu.co/handle/11634/30031> [consulta: 4 de febrero de 2021].

GEHL, Jan (2003). La humanización del espacio urbano: la vida social entre edificios (M. ${ }^{\mathrm{a}}$ T. Valcarce, trad.). Barcelona: Reverté, 2017.

GHARBI, Ibrahin y KNIGHT, Alan (2017). «L'émergence et l'implantation du centre commercial régional dans la ville nord-américaine». Ateliers d'Anthropologie, 44, 1-21. <https://doi.org/10.4000/ateliers.10368>

GidDENS, Anthony (2007). Europa en la Era Global (A. Santos, trad.). Barcelona: Paidós.

Gladwell, Malcolm (2004). «The terrazzo jungle». The New Yorker, 15, 120-127.

GöBEL, Christof (ed.) (2016). Espacio público, aprendiendo en la plaza urbana. Ciudad de México: Universidad Autónoma de México.

GonZÁlez AguirRe, Raúl R. (2017). «La relación entre consumo y espacio público. El caso de los centros comerciales en la zona metropolitana de Monterrey». Humanitas, 44 (2), 151-178.

GONZÁLEZ ESCOBAR, Luis F. (2010). "Los centros comerciales o el simulacro del espacio público». Agenda Cultural, 168, 1-7.

Gorelik, Adrián y Silvestri, Graciela (1991). «Postales de Buenos Aires (notas para leer en el subte)». Nueva Sociedad, 114, 124-132.

Goss, Jon (1993). "The "magic of the mall": An analysis of form, function, and meaning in the contemporary retail built environment». Annals of the Association of American Geographers, 83 (1), 18-47. <https://doi.org/10.1111/j.1467-8306.1993.tb01921.x>

Gosseye, Janina y Avermaete, Tom (2019). «Introduction. A malleable type: acculturating the shopping centre». En: Gosseye, Janina y AVERMAETE, Tom (eds.). Acculturating the Shopping Centre. Londres: Routledge, 1-17.

Gruen, Victor y SMITH, Larry (1960). Shopping Towns USA: The Planning of Shopping Centers. Nueva York: Van Nostrand Reinhold. 
Hardwick, M. Jeffrey (2004). Mall Maker: Victor Gruen, Architect of an American Dream. Filadelfia: University of PA Press.

HarveY, David (2008). «La libertad de la ciudad». Antípoda, 7, 14-30. <https://doi.org/10.7440/antipoda7.2008.02>

HeidegGer, Martin (1994). Conferencias y artículos. Barcelona: Ediciones del Serbal.

Higueras Arnal, Antonio M. (2003). Teoría y método de la Geografía. Introducción al análisis geográfico regional. Zaragoza: Prensas Universitarias de Zaragoza.

Howeln, Philip (1993). "Public space and the public sphere: political theory and the historical geography of modernity». Environment and Planning D: Society and Space, 11 (3), 303-322. $<$ https://doi.org/10.1068/d110303>

Iso TinOCO, Andoni (2009). «Los centros comerciales del área metropolitana de Pamplona, cambio y reestructuración». Zainak, 31, 257-273.

JACKSON, Kenneth T. (1996). "All the world's a mall: Reflections on the social and economic consequences of the American shopping center». The American Historical Review, 101 (4), 1.111-1.121. <https://doi.org/10.2307/2169636>

Jackson, Vanessa; STOel, Leslie y Brantley, Aquia (2011). «Mall attributes and shopping value: Differences by gender and generation cohort». Journal of Retailing and Consumer Services, 18 (1), 1-9.

<https://doi.org/10.1016/j.jretconser.2010.08.002>

JACOBS, Allan B. (1993). Grandes Calles (Gabinete de Traducción Babel, trad.). Santander: Universidad de Cantabria, 1996.

Jiménez Domínguez, Bernardo; Becerra Mercado, Olga y Olivera, Ana R. (2009). «Apropiación pública del espacio en centros comerciales de la Zona Metropolitana de Guadalajara». Medio Ambiente y Comportamiento Humano, $10(3), 253-285$.

Kitchin, Rob y TaTe, Nicholas J. (2000). Conducting Research into Human Geography, Theory, Methodology \& Practice. Harlow: Prentice Hall.

Klein, Naomi (1999). No logo (A. Jockl, trad.). Barcelona: Paidós, 2001.

KoHn, Margaret (2004). Brave New Neighbourhoods. The Privatization of Public Space. Londres: Routledge.

KoOHSARI, Mohammad J.; Mavoa, Suzanne; Villanueva, Karen; SugIYama, Takemi; Badland, Hannah; KaCZynski, Andrew T.; Owen, Neville y Giles-Corti, Billie (2015). «Public open space, physical activity, urban design and public health: Concepts, methods and research agenda». Health \& Place, 33, 75-82. <https://doi.org/10.1016/j.healthplace.2015.02.009>

KowINSKI, William S. (2002). The Malling of America. Travels in the United States of Shopping. Bloomington: Xlibris Corporation.

Lefebvre, Henri (1968). El derecho a la ciudad (I. Martínez y J. González-Pueyo, trads.). Madrid: Capitán Swing, Madrid, 2017.

- (1974). La producción del espacio (P. M. Lorea y E. Martínez, trads.). Madrid: Capitán Swing, 2013.

LÓPEZ LEVI, Liliana (1999). Centros comerciales: espacios que navegan entre la realidad y la ficción. Ciudad de México: Nuestro Tiempo.

Low, Setha M. (2006). «The erosion of public space and the public realm: paranoia, surveillance and the privatization in New York City». City \& Society: Journal of the Society for Urban Anthropology, 18 (1), 43-49. <https://doi.org/10.1525/city.2006.18.1.43> 
Low, Setha M. y Smith, Neil (2006). The Politics of Public Space. Nueva York: Routledge.

Lulle, Thierry y PAQUeTTE, Catherine (2006). «Los grandes centros comerciales y la planificación urbana. Un análisis comparado de dos metrópolis latinoamericanas». Estudios Demográficos y Urbanos, 22 (2), 337-361.

Madanipour, Ali (1996). Design of Urban Space. Nueva York: Wiley.

Maderuelo, Javier (2005). El paisaje. Génesis de un concepto. Madrid: Adaba.

Marín de la CruZ, Sara y Martín Cerdeño, Víctor J. (2013). "Centros comerciales en España. Situación, evolución e interpretación empírica». Revista Distribución y Consumo, 5, 5-23.

Medina CANO, Federico (1998). "El centro comercial: una "burbuja de cristal”". Estudios sobre las Culturas Contemporáneas, 4 (8), 61-91.

Michon, Richard; Yu, Hong; Smith, Donna y Chebat, Jean-Charles (2008). «The influence of mall environment on female fashion shoppers' value and behaviour». Journal of Fashion Marketing and Management, 12 (4), 456-468.

Miller, Jacob C. y LAKETA, Sun ana (2018). "The "magic of the mall" revisited: Malls and the embodied politics of life». Progress in Human Geography, 43 (5), 910-926. <https://doi.org/10.1177/0309132518794274>

Mitchell, Don (2003). The Right to the City. Social Justice and the Fight for Public Space. Nueva York: The Guilford Press.

Molina CALDERÓn, Jennifer (2019). «De la experiencia religiosa a la experiencia del consumo en asistentes a misas en tres centros comerciales de Bogotá». XXII Congreso Internacional Alas Perú 2019. Recuperado de <https:/www.researchgate.net/ publication/338434838_De_la_experiencia_religiosa_a_la_experiencia_del_consumo_en_asistentes_a_misas_en_tres_centros_comerciales_de_Bogota $>$ [consulta: 4 de febrero de 2021].

MonneT, Jérôme (1996). «Espacio público, comercio y urbanidad en Francia, México y Estados Unidos». Alteridades, 6 (11), 11-25.

Moroni, Stefano y CHIOdelli, Francesco (2013). «The relevance of public space: Rethinking its material and political aspects». En: BASTA, Claudia y MORONI, Stefano (eds.). Ethics, design and planning of the built environment. Berlín: Springer, 45-55.

Moulian, Tomás (1999). El consumo me consume. Santiago de Chile: Libros del Ciudadano.

Nahavandi, Elham; Zabetian, Elham; Ahmad, Ahmad P. y Kheyroddin, Reza (2017). "Spaces' livability in modern commercial centers». Manzar, the Scientific Journal of Landscape, 9 (40), 48-59.

NÉMETH, Jeremy (2009). «Defining a public: The management of privately owned public space». Urban Studies, 46 (11), 2.463-2.490. <https://doi.org/10.1177/0042098009342903>

NoguÉ, Joan (ed.) (2007). La construcción social del paisaje. Madrid: Biblioteca Nueva. OrTiz, Renato (2004). Mundialización y Cultura. Bogotá: Convenio Andrés Bello.

PÁramo, Pablo (2011). Sociolugares. Bogotá: Universidad Piloto de Colombia.

PARLeTte, Vanessa y Cowen, Deborah (2011). «Dead malls: suburban activism, local spaces, global logistics». International Journal of Urban and Regional Research, 35 (4), 794-811. <https://doi.org/10.1111/j.1468-2427.2010.00992.x>

Peñalosa, Enrique (2017). «Prólogo». En: GeHL, Jan. La humanización del espacio urbano: la vida social entre edificios. Barcelona: Reverté, 7-8. 
PinTAudi, Silvana M. y FrúgOLI, Heitor (1992). Shopping centers: espaço, cultura e modernidade nas cidades brasileiras. São Paulo: UNESP.

QIAN, Junxi (2020). «Geographies of public space: Variegated publicness, variegated epistemologies». Progress in Human Geography, 44 (1), 77-98. $<$ https://doi.org/10.1177/0309132518817824>

RAO, Fujie; DOVEY, Kim y PAFKA, Elek (2017). «Towards a genealogy of urban shopping: Types, adaptations and resilience». Journal of Urban Design, 23 (4), 544-557. <https://doi.org/10.1080/13574809.2017.1405726>

Ravetllat, Pere Joan y Ribas, Carme (2019). "Nuevo espacio público en el entorno del Mercado de Sant Antoni (Barcelona)». Planur-e, 13, 1-10.

Ritzer, George (1994). La McDonalización de la sociedad (I. Hierro y R. Hierro, trads.). Barcelona: Ariel, 1996.

RoCHMINSKA, Agnieszka (2017). «Shopping centres as elements of the functional and spatial structures of cities. Location, impact, change tendencies, development perspectives». Barometr Regionalny, 15 (2), 55-65.

SACK, Robert D. (1988). «El lugar y su relación con los recientes debates interdisciplinarios». Documents d'Anàlisi Geogràfica, 12, 223-241.

SAlCEDO, Rodrigo (2002). «El espacio público en el debate actual: una reflexión crítica sobre el urbanismo post-moderno". EURE, 28 (84), 5-19.

- (2003). "When the global meets the local at the mall». The American Behavioral Scientist, 46 (8), 1.084-1.103. <https://doi.org/10.1177/0002764202250500>

Santos, Milton (1988). Metamorfosis del espacio habitado (G. M. Vargas, trad.). Barcelona: Oikos Tau, 1996.

- (1994). Por otra globalización. Del pensamiento único a la conciencia universal (G. Guerrero, trad.). Bogotá: Convenio Andrés Bello, 2004.

- (1997). La naturaleza del espacio. Técnica y tiempo. Razón y emoción (M. L. Silveira, trad.). Barcelona: Ariel, 2000.

SARLO, Beatriz (1998). «El centro comercial». La Jornada Semanal. Recuperado de <https://www.jornada.com.mx/1998/03/22/sem-sarlo.html> [consulta: 7 de septiembre de 2020].

- (2009). La ciudad vista. Mercancías y cultura urbana. Buenos Aires, Argentina: Siglo XXI.

SASSANO LuIZ, Silvana F. (2015). "Imagen, localización y evolución de los centros comerciales en Argentina: un estudio de caso (Buenos Aires)». Documents d'Anàlisi Geogràfica, 61 (2), 409-432. <https://doi.org/10.5565/rev/dag.238>

Sassen, Saskia (1991). La ciudad global. Nueva York, Londres, Tokio. Buenos Aires: Eudeba, 1999.

SerJe, Margarita y SAlcedo, Andrés (eds.) (2008). «Antropología del espacio: etnografías del paisaje y del lugar». Antípoda, 7, 11-320.

Serrano López, Augusto y Stein Heinemann, Alfredo (2019). Re-construyendo la ciudad. El espacio público como lugar de simetría, diálogo y transcendencia. Alicante: Universidad de Alicante.

SHIELDS, Rob (1992). «Spaces for the subject of consumption». En: SHIELDS, Rob (ed.). Lifestyle shopping: The subject of consumption. Londres: Routledge, 1-20.

Silva GraçA, Miguel (2018). "The new (public) space: On cities shopping centers demonstrations and the future of public uses». Journal of Communication and Languages, 48, 191-207. 
Simone, Rosa L. de (2018a). «The Latin American shopping centre: Cultural translation, symbolic adaptation and typological evolution of commercial architecture in Latin American cities». En: Gosseye, Janina y Avermaete, Tom (eds.). Acculturating the Shopping Centre. Londres: Routledge, 64-90.

- (2018b). «Instalando la ciudad del consumo: el palimpsesto urbano del primer shopping mall chileno en el fundo San Luis, Santiago». EURE, 44 (133), 91-112.

- (2019). «Festival malls». The Wiley Blackwell Encyclopedia of Urban and Regional Studies.

<https://doi.org/10.1002/9781118568446.eurs0101>

SKLAIR, Leslie (1991). Sociology of the global system. Nueva York: Harvester Wheatsheaf.

- (2010). «Iconic architecture and the culture-ideology of consumerism». Theory, Culture and Society, 27 (5), 135-159. <https://doi.org/10.1177/0263276410374634>

SojA, Edward W. (1989). Postmodern Geographies. The Reassertion of Space in Social Theory. Londres: Verso.

SPRINGER, Simon (2011). «Public space as emancipation: Meditations on anarchism, radical democracy, neoliberalism and violence». Antipode, 43 (2), 525-562. <https://doi.org/10.1111/j.1467-8330.2010.00827.x>

StaeHeli, Lynn A. y Mitchell, Don (2007). «Locating the public in research and practice». Progress in Human Geography, 31 (6), 792-811. <https://doi.org/10.1177/0309132507083509>

STYHRE, Alexander (2019). "The invention of the shopping mall: Victor Gruen and production of the high-liquidity, capitalist space». Journal of Engineering, Design and Technology, 17 (2), 283-299.

<https://doi.org/10.1108/jedt-08-2018-0139>

SUSSER, Ida (ed.) (2001). La sociología urbana de Manuel Castells. Madrid: Alianza.

TCHOUKAlEyska, Roza; EnRIght, Theresa y LeHrer, Ute (2019). "Public space beyond the city centre: Suburban and periurban dynamics». Canadian Journal of Urban Research, 28 (1), 1-4.

Tester, Keith (1994). The Flâneur. Londres: Routledge.

Thaler, Richard H. y Sunstein, Cass R. (2003). "Libertarian paternalism». The American Economic Review, 93 (2), 175-179. <https://doi.org/10.1257/000282803321947001>

THrifT, Nigel (2008). Non-Representational Theory: Space, Politics, Affect. Nueva York: Routledge.

Tiмотнy, Dalle J. (2005). Shopping Tourism, Retailing and Leisure [eBook]. Channel View Publications.

TOMÉ FERNÁNDEZ, Sergio (2014). «Espacios públicos singulares en áreas urbanas centrales». Ciudad y Territorio, XLVI (180), 277-290.

TORABI, Elnaz; DeDEKOrKuT-Howes, Aysin y Howes, Michael (2021). «A framework for using the concept of urban resilience in responding to climate-related disasters». Urban Research \& Practices. <https://doi.org/10.1080/17535069.2020.1846771>

Underhill, Paco (2004). Call of the Mall [eBook]. Simon \& Schuster.

Valdelvira Vega, Míriam (2010). «Escudero Gómez, Luis Alfonso. Los centros comerciales, espacios postmodernos de ocio y consumo: un estudio geográfico». Documents d'Anàlisi Geogràfica, 56 (3), 636-641.

VERDÚ, Vicente (1996). El planeta americano. Barcelona: Anagrama.

VILAS, Manuel (2017). América (3. ${ }^{a}$ edición). Madrid: Círculo de Tiza. 
VOYCE, Malcolm (2006). «Shopping malls in Australia: the end of public space and the rose of "consumerist citizenship"?». Journal of Sociology, 42 (3), 269-286. <https://doi.org/10.1177/1440783306066727>

YE, Junjia (2019). "Re-orienting geographies of urban diversity and coexistence: Analyzing inclusion and difference in public space». Progress in Human Geography, 43 (3), 478-495. <https://doi.org/10.1177/0309132518768405>

Zuleta Ruiz, Beethoven (2013). Territorio y catolicismo en Colombia: Antioquia (siglos $X V I I-X X)$. Bogotá: Universidad Nacional de Colombia. 
